\title{
Optimization Analysis of Cooling Process of 3D-Printing in FDM
}

\author{
Li yanxiang $^{1, a^{*}}$, Cao Guangchun ${ }^{1, b}, X_{i e} L^{1, c}$ and Huang Dahai ${ }^{2, d}$ \\ ${ }^{1}$ College of Hydraulic and Environmental Engineering, China Three Gorges University, Yichang, \\ 443000, Hubei, China \\ ${ }^{2}$ School of Transportation Science and Engineering, Beihang University, Beijing, 100191, China \\ aliyanxiang330@126.com, bcaoguangchun5023@163.com, ${ }^{\mathrm{c}} 245634255 @ q q . c o m$, \\ dhuangdh@tsinghua.edu.cn
}

Keywords: Fused deposition modeling (FDM), model dimension, cooling condition, thermal stress

\begin{abstract}
Fused deposition modeling (FDM) has brought great innovation for manufacturing because of its simple processing and forming rapidly, but the printing products created by this way are always found low-strength, deformed, cracked and so on. Aiming at these problems, there have been many researches on the development of new materials and the optimization of process parameters, while the after treatment, particular the cooling process are ignored. In this paper, the thermal stress in different-dimension and different-material models are analysised and the relationship between thermal stress and different cooling conditions are explored. The result demonstrates the actual reason of that the bigger, the easier to crack, and find that adjusting the environment temperature to multistep decline can reduce the thermal stress obviously.
\end{abstract}

\section{Introduction}

With the development of rapid prototyping, 3D printing has gradually been widely used. Compared with the traditional manufacturing technique, 3D printing has irreplaceable advantages: high speed, energy saving and the infinite space for design.

Depending on the different techniques, 3D printing is divided into more than ten kinds of production methods, such as Stereo Lithography Appearance (SLA), Selective Laser Sintering (SLS), Fused Deposition Modeling (FDM) and so on, among which FDM is widely used in desktop 3D printer. FDM is a kind of printing process, which transports filiform material into the nozzle and heats to melt, then deposits the melt material on the workbench according to section information, which can form expected product layer-by-layer.

There are still some defects in the manufacturing process of 3D printing model, such as the existing of residual stress and localdeformation, which is mostly because the uneven and synchronized energy input, and can effect low dimensional and form accuracy, low structure strength and even crack.

Many researchers have always been in search of the solution of these problems, Fuda Ning et al. [1] investigated adding reinforced materials into plastic materials to form thermoplastic matrix carbon fiber reinforced plastic (CFRP) and improve the mechanical properties of FDM-fabricated parts. O.S. Carneiro et al. [2] found that the thickness of the layers has little influence on the mechanical performance of the samples, the infill degree has a dramatic and linear effect on the mechanical properties and the use of fibers as reinforcement is also effective in $3 \mathrm{D}$ printing. L. Villalpando et al [3] developed a model as a predictive tool to get optimization model that considers the build time, material usage, surface finish, interior geometry, strength characteristics and so on, which can be used to the assist design of optimal combination. You-Min Huang et al.[4] obtained a new CAD model by means of reverse distortion compensation and sent it to a rapid prototyping machine for the actual prototyping processes, so as to obtain a more accurate precision. Start Singamneni et al.[5] proposed the curved layer deposition for FDM, in particular for thin shell-like parts, to ensure fibre continuity, then printed a few models by this way, and found marked improvement in the mechanical characteristics of curved layer parts. Antreas Kantaros and Dimitris Karalekas[6] investigates the 
magnitude of the solidification induced residual strains in FDM fabricated parts using different processing parameters, particular the layer thickness and the deposition orientation.

The most of the research is mainly focused on the optimization of process parameters and the development of new material, while ignores the post-processing, particular the effect of temperature variation. This paper investigated the relationship among the dimension, cooling condition and thermal stress of $3 \mathrm{D}$ printing models, and looked for the approach to reduce thermal stress.

\section{Model information}

The kinds of available materials used in desktop 3D printer are limited, and ABS (Acrylonitrile Butadiene Styrene), PLA ( Poly Lactic Acid) and POM (polyformaldehyde) are widely used at present. Calculation model are based on ABS and POM, while the material of work plane is glass.

In order to prevent the model leaving or destroying the work plane, there is always high temperature tape pasted on the platform, which is ignored in this simulated analysis and replaced by all DOF at the base of the model.

The mold temperature of $\mathrm{ABS}$ and $\mathrm{POM}$ is about $170^{\circ} \mathrm{C}$. In the real manufacture process, the cooling condition is not strictly limited, and the usually practice is room temperature directly, some products with unusual requirements should be maintained in drying oven with constant temperature at $70^{\circ} \mathrm{C}-80^{\circ} \mathrm{C}$. At this section set $70^{\circ} \mathrm{C}$ as cooling condition

Take a quarter model for the simulation calculation, and there are a total of three models:

A: The model dimension is $0.02 * 0.016 * 0.08 \mathrm{~m}$, and the platform's is $0.04 * 0.04 * 0.015 \mathrm{~m}$;

$\mathrm{B}$ : The model dimension is $0.1 * 0.08 * 0.4 \mathrm{~m}$, and the platform's is $0.25 * 0.25 * 0.06 \mathrm{~m}$;

The geometry is shown in Fig.1. In order to explore displacement and stress of model more intuitively, there are five typical points are selected, and the distribution is shown in Fig.2.

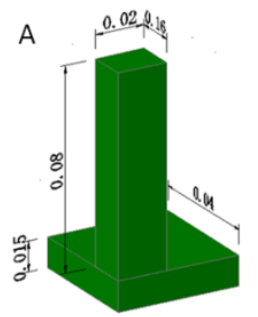

Fig.1 Model dimension
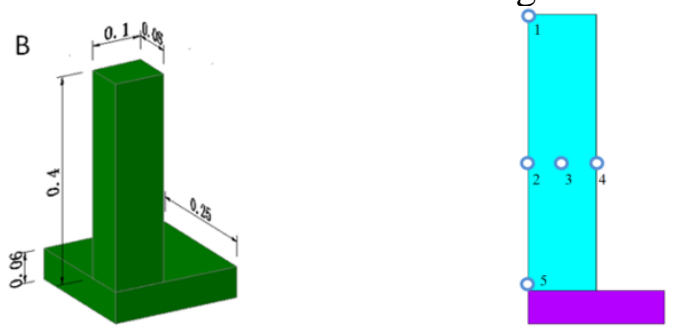

Fig.2 Points position

\section{Interpretation of result}

The major principal stress and the Z displacement in typical point 1 of ABS and POM are presented in Fig. 3 and 4 . The results show that there are the similar trends about the thermal stress and displacement of two materials changing along with time, and thermal stress which reduced gradually and tends to be stable after the peak is closely relative to the model size; the shrinkage deformation and thermal stress of ABS are all much smaller than the POM, and in the process of cooling, the max stress of model A, B created by ABS is 3.54 $\mathrm{MPa}$ and 15.77 MPa respectively, while 4.87MPa and 21.33 MPa for POM.

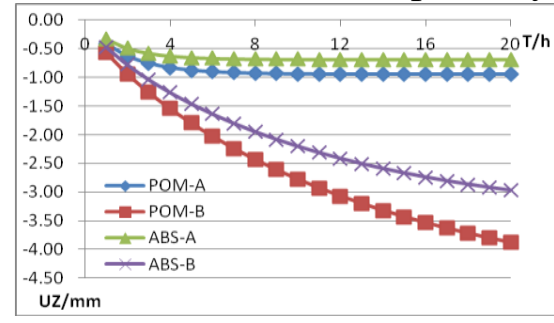

Fig.3 UZ of point 1

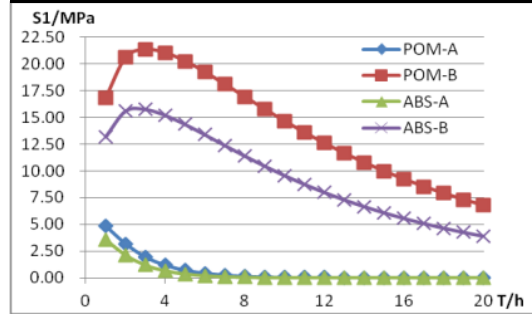

Fig.4 S1 of point 1

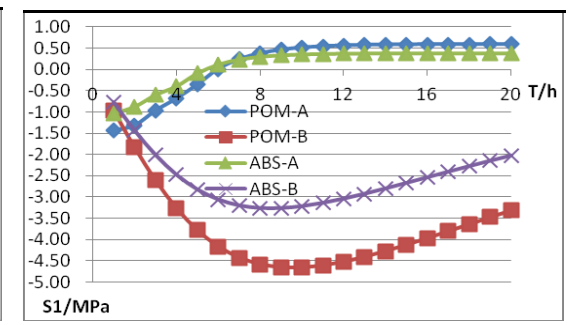

Fig.5. S1 of point 2

Typical points 2 and 3 are located inside the model, the major principal stress in which are presented in Fig.5 and 6. The result shows that, there are the similar trends about the thermal stress and displacement of 
two materials changing along with time, and thermal stress which reduced gradually and tends to be stable after the peak is closely relative to the model size.

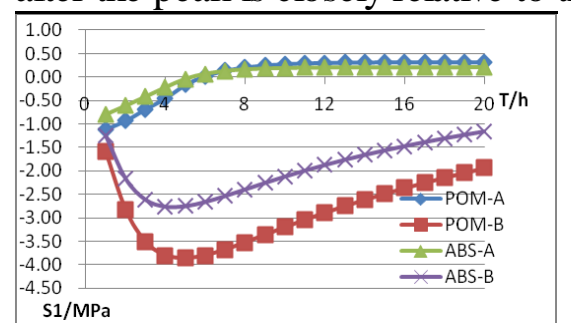

Fig.6. S1 of Point 3

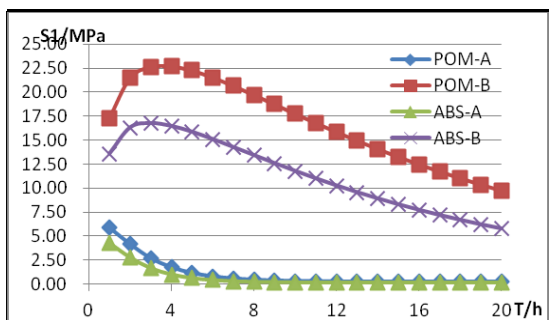

Fig.7. S1 of Point 4

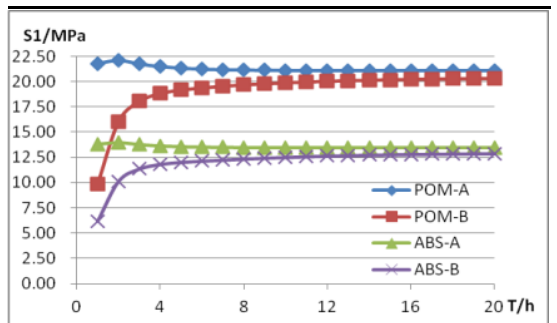

Fig.8. S1 of Point 5

The major principal stress in point 4 and 5 of ABS and POM are presented in Fig. 7 and 8. Point 4 is located at the boundary, which contacts with the outside temperature directly. According to Fig.7, in the cooling process, the bigger the model is, the higher the thermal stress will be, and the thermal stress reduces along with time and trends to be 0 after the peak. Point 5 belongs to the bottom of the center, and different from point 4 , the stress of point 5 keeps the same after reaching peak and the stable value is very close no matter the model is big or not. These results is due to a bond between material and work plane in the phase change process, which results in the bottom restrained by bonding surface when cooling shrinkage, and unable to change shape along with time freely. This kind of restraint stress can be reduced only after the model breaking away from the work plane.

\section{The optimization of cooling process}

Set model with ABS material, take the B model for calculation model, and investigate the deformation degree and changing process of thermal stress when the environment temperature changes ladder likely over time in the model cooling process.

Setting for cooling condition. There are 4 kinds of cooling conditions: A: The outside temperature is $20^{\circ} \mathrm{C}$ all the time; B: Environmental initial temperature is $170^{\circ} \mathrm{C}$, cooling rate is $20^{\circ} \mathrm{C} / \mathrm{h}$, and maintain constant after $20^{\circ} \mathrm{C}$; C: Environmental initial temperature is $170^{\circ} \mathrm{C}$, cooling rate is $10^{\circ} \mathrm{C} / \mathrm{h}$, and maintain constant after $20^{\circ} \mathrm{C}$; D: Environmental initial temperature is $170^{\circ} \mathrm{C}$, cooling rate is $4^{\circ} \mathrm{C} / \mathrm{h}$, and maintain constant after $20^{\circ} \mathrm{C}$ 。

\section{The stress analysis of optimization of cooling process.}

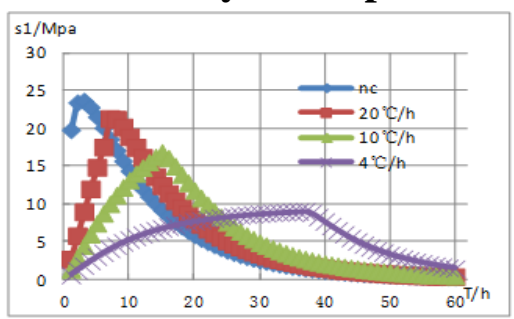

Fig.9. $\mathrm{S} 1$ of point 1

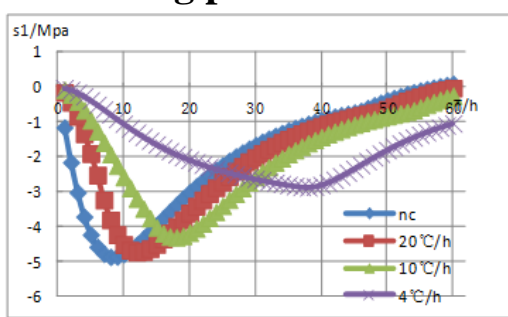

Fig.10 S1 of point 2

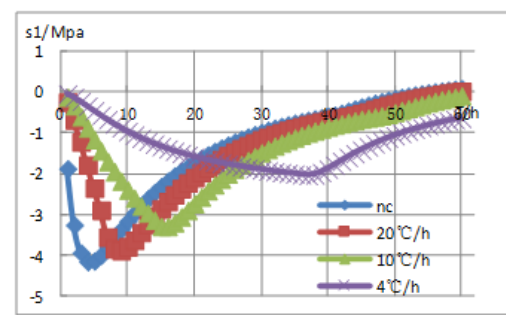

Fig.11 S1 of point 3

It is shown in Fig.9 that the thermal stress is tensile stress at the upper surface point, which tends to be 0 with time increasing, and the maximum stress gradually decreases with the slowing down of cooling rate.

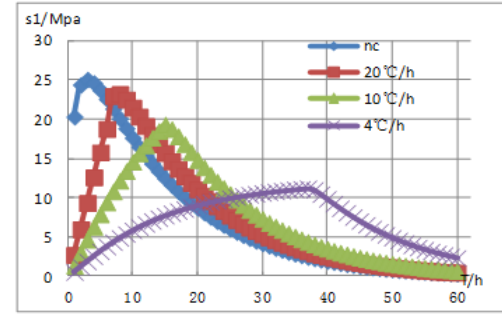

Fig. 12 S1 of point 4

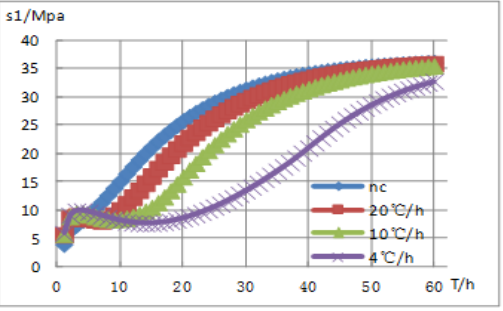

Fig. $13 \mathrm{~S} 1$ of point 5

Point 2, 3 and 4 are all at the middle elevation, but as a boundary point, the change trend of the first principal stress of point 4 is similar to point 1 , namely the thermal stress is tensile stress, and tends to 0 gradually. Except for the point 4 , the rest two points are all in compressive stress, and the maximum stress 
reduces gradually with the cooling rate slowing down. At point 5 in Fig. 13 the cooling rate has nothing to do with the thermal stress, which will eventually tend to be the same larger non-zero value, as a result, it is important to select the stripping time.

The maximum value of first principal stress of various typical points in model B is shown in table 1 .

Table 1. Maximum major principal stress of model B (MPa)

\begin{tabular}{|c|c|c|c|c|c|}
\hline \multirow{2}{*}{$\begin{array}{c}\text { Cooling } \\
\text { Rate }\end{array}$} & \multicolumn{5}{|c|}{ Num Number } \\
\cline { 2 - 6 } & $\mathbf{1}$ & $\mathbf{2}$ & $\mathbf{3}$ & $\mathbf{4}$ & $\mathbf{5}$ \\
\hline $\mathrm{nc}$ & 23.7 & -4.9 & -4.2 & 25.1 & 36.1 \\
\hline $20 \mathrm{C}^{\circ} / \mathrm{h}$ & 21.3 & -4.7 & -3.9 & 23.3 & 35.9 \\
\hline $10 \mathrm{C}^{\circ} / \mathrm{h}$ & 16.8 & -4.3 & -3.3 & 19.4 & 35.5 \\
\hline $4 \mathrm{C}^{\circ} / \mathrm{h}$ & 9 & -2.9 & -2 & 11.3 & 32.8 \\
\hline
\end{tabular}

It is more intuitive in table 1 that there will be more places with higher thermal stress than the allowable stress of $\mathrm{ABS}$ material when the model is put in the environment of $20{ }^{\circ} \mathrm{C}$ directly, while the maximum of thermal stress can be reduced significantly when adjusting environmental temperature from the product molding temperature down to room temperature little by little, and the lower the cooling rate of environment is, the lower the maximum thermal stress is.

As a result, the optimization of cooling process can do help with the material with high mechanical performance requirements, as to the material with high accuracy requirements, another technological means may make sense, such as compensation[4], hybrid rapid prototyping system and so on.

\section{Conclusions}

The effect of cooling condition on the thermal stress of model was investigated in this research. The following conclusions can be deduced from the study:

1. Different materials, different shrinkage and inner stress. ABS model has higher accuracy than POM model.

2. In order to prevent high restraint stress appears at the bottom of the model, the model should be stripped as soon as it reached intensity.

3. The bigger the model is, the higher the thermal stress in the cooling process will be, therefore it can't be ignored to optimize model dimension on the basis of meeting the product performance. As for the model cannot be optimized, developing the performance of material can do some help.

4. Pay attention to the post processing, particular the cooling process. The reasonable control of environmental cooling rate can reduce the thermal stress of model and improve the performance.

5. It still needs to use other means of optimization on the basis of adjusting cooling process for the product with high accuracy requirement.

\section{Reference}

[1] Fuda Ning, Weilong Cong, Jingjing Qiu, Junhua Wei, Shiren Wang, Additive manufacturing of carbon fiber reinforced thermoplastic composites using fused deposition modeling, Composites Part B 80 (2015) 369-378.

[2] O.S. Carneiro, A.F. Silva, R. Gomes, Fused deposition modeling with polypropylene, Materials \& Design 83 (2015) 768-776.

[3] L. Villalpando, H. E iliat, R. J. Urbanic, An optimization approach for components built by fused deposition modeling with parametric internal structures, Procedia CIRP 17 (2014) 800-805.

[4] You-Min Huang, Hsiang-Yao Lan, Compensation of distortion in the bottom exposure of stereolithography proces, Int J Adv Manuf Technol,2006,27:1101-1112.

[5] Sarat Singamneni, Asimava Roychoudhury, Olaf Diegel, Bin Huang, Modeling and evaluation of curved layer fused deposition, Journal of Materials Processing Technology 212 (2012) 27- 35.

[6] Antreas Kantaros, Dimitris Karalekas, Fiber Bragg grating based investigation of residual strains in ABS parts fabricated by fused deposition modeling process, Materials and Design 50 (2013) 44-50. 